\title{
Tag4M in Predictive Maintenance and Machine Control
}

\author{
doi:10.3991/ijoe.v6i1.1206 \\ D. Ursutiu ${ }^{1}$, M. Ghercioiu ${ }^{2}$, R. Oros ${ }^{1}$ and C. Samoila ${ }^{1}$ \\ ${ }^{1}$ University “Transylvania”, Brasov, Romania \\ 2 TAG4M, Austin, USA
}

\begin{abstract}
This paper tray to introduce in the field of Predictive Maintenance and System Monitoring a new devices the Tag4M. This Wi-Fi tag has been built by Tag4M of Austin, TX, USA, with the purpose of connecting sensors to the Internet where data is displayed using Web Pages that are posted and can be accessed from anywhere in the world. The Tag4M Wi-Fi tag is tiny and low cost system working on battery power, and therefore it is very suitable for monitoring and reliability estimation of expensive systems.
\end{abstract}

Index Terms-Maintenance, Machine Control, Active TAG's, Wireless DAQ, Monitoring.

\section{INTRODUCTION}

There are some progresses in fabrication which have imposed the transfer from the keep up towards maintenance. The stronger ones there is development of the nondestructive control methods appearance which allows the introduction of the first stage of the maintenance processdiagnosis. The diagnosis process, together with the reliability and risk evaluation methods, has generated two philosophies regarding maintenance:

Life Cycle Cost (LLC) which has as main objective the minimization of the exploitation costs;

Total Productive Maintenance (TPM) which, apart of the economic approach, has added the human resources role.

Our Tag4M system there is a component of the technological aspects of the maintenance process, used for supervision and diagnose of the functionality of equipments apart of the human subjectivism.

If we are able to define with certainty that, in the functionality period of the equipment $T, F(t)$ the spoil probability will be valuable for maintenance cost the expression:

$$
C_{M S}=C_{d m}+C_{I} \cdot F(t)
$$

where:

$C_{M S}$ - the total probable cost of the systematic maintenance operations;

$C_{d m}$ - the direct cost of the maintenance activities;

$C_{I}$ - the indirect costs, including those of the unavailability of equipment;

The above expression is referred at the case in which the defective previsions are inside of the time interval $T$.
If the accidental defection will occur before of this time interval, the costs will increase proportional:

$$
C_{T m}(t)=\frac{C_{d m}+C_{I} \cdot F(t)}{m(t)}
$$

The optimum period of functionality, between two successive overhauling, will be obtained from the condition that $C_{\operatorname{Tm}}(t)$ to be minim.

Above relations are valuable in the case of the systematic maintenance. In the case of the preventive maintenance will be added the cost of the measurement and control equipment which help us to supervise and to prevent.

$$
C_{M P}=\frac{C_{d m}+C_{a m c}}{\Psi \cdot \frac{m(t)}{\Psi}}
$$

where: $C_{\text {amc }}$ there is the cost of the implementation of the preventive maintenance:

$$
C_{\text {amc }}=\left[\frac{A_{c h}}{D_{a m}} \cdot \frac{m(t)}{\Psi}\right]+C_{c}
$$

$A_{c h}$ - the costs of the measurement and control equipments used at supervision;

$D_{a m}$ - the estimated period for measurement and control equipments acquisition;

$C_{C}$ - the cost of the measurement and control operations on the interval $T_{m b f}=\frac{m(t)}{\Psi}$;

All of the above considerations give to us the idea that the quality of maintenance is in strong dependence with the applied method, but in the mean time the idea that, preventive maintenance which employed the measurement and control devices is only one which allows the estimation with high certainty the period of good functionality. If we accept this, the rest of the discussions are oriented towards the cost of the supervision equipments.

Tag4M (Figure 1) is the cheaper and the smaller equipment which fulfil at high level the measurement and control operations. It will open a new era in the maintenance. 


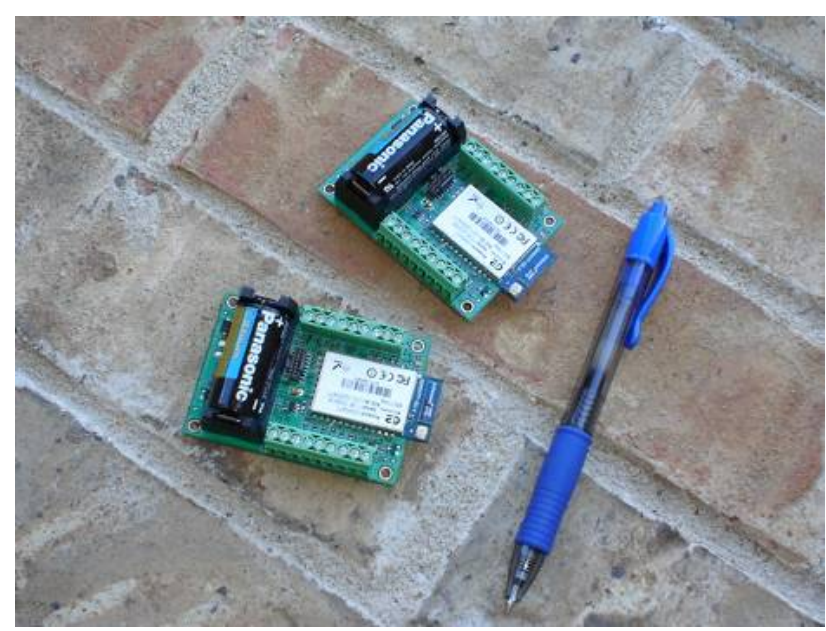

Figure 1. Tag4M Device

\section{TAG4M MONITORING}

\section{A. Tag4m - The Wireless Device}

Knowing when a critical plant component is headed towards a breakdown means that plant engineers can save facility considerable money. Predictive analysis can also detect an upcoming malfunction that might not be revealed in a routine maintenance schedule, allows users to track and identify potential problems, verify new installations and establish baselines for future inspections.

A key tool for predictive maintenance is vibration analysis because the more complex the machinery, the more it "talks" to us - but at frequencies our ears often cannot hear and in a language we must learn. Consider your family car. When you turn the ignition and drive it down the street, you are greeted with familiar sounds of the engine, breaks, tires and other systems. We all know the feeling of "something doesn't sound right today" and try to isolate the problem before it leads to danger to ourselves or our families.

To do the same for complex machinery, plant engineers place vibration sensors at key locations on the equipment to monitor moving parts such as rollers, bearing, pumps, universals, couplings, etc. Over time, these engineers can build up a database of familiar vibration patterns and thus more easily spot deviations from expected patterns.

One problem, though, is the cost and method of attaching sensors and their cabling on very large machines or those that are constantly in motion, such as an overhead crane.

This is where the Tag $4 \mathrm{M}$ is an ideal solution because many factories are today equipped with wireless hot spots, and given off-the-shelf technology it is inexpensive to add this functionality to those that are not so equipped. The Tag4M is unique in its ability to place measurement data into a web environment that does not depend on any single computer. The tag sends data to an off-the-shelf Access Point (AP) which routes the data to the Internet for analysis, modeling, simulation, and presentation on web pages. The AP can also route measurement data to computers located in a local LAN.

\section{B. Sensors and Software}

Accelerometers are the preferred motion sensors for most vibration monitoring applications, and the Tag4M interfaces to standard units such as the ADXL330 from Analog Devices with only a few external signalconditioning components. In the Figure 2 we present the motion sensor and the Tag4M connectivity.

This Tag4M system can be controlled in National Instruments Graphical Programming software LabVIEW. We developed the following LabVIEW application that can import data from such an acceleration sensor and display its values (see the Figure 3 ).

In its current implementation, the Tag4M samples at a maximum rate of $3.3 \mathrm{~Hz}$, which is adequate for some but not most vibration-monitoring applications? In these other cases, this low-frequency approach can serve as a proof of concept. Then, when the end user has completely defined project requirements, he can work with the supplier to create a customized tag that is populated with the accelerometer directly on the tag (eliminating the need for an outboard accessory module) and without the conventional jumper blocks, thus reducing overall size and cost. This approach can also implement channel scanning, which provides for sampling rates to $30 \mathrm{kHz}$ with readings being stored locally in tag EEPROM between connections with a wireless access point.

The tag also lets users place measurement circuitry immediately adjacent to the physical process and removes worries about cabling such as noise they are subject to and costs. In addition, each tag can function as an embedded machine controller thanks to its on-board intelligence (32bit RISC processor), EPROM, RAM (64k bytes) and 4

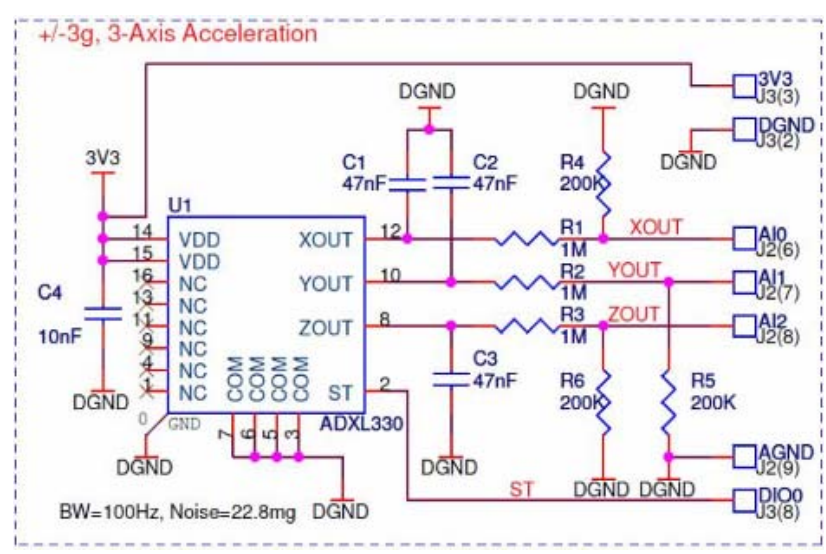

Figure 2. The Motion Sensor

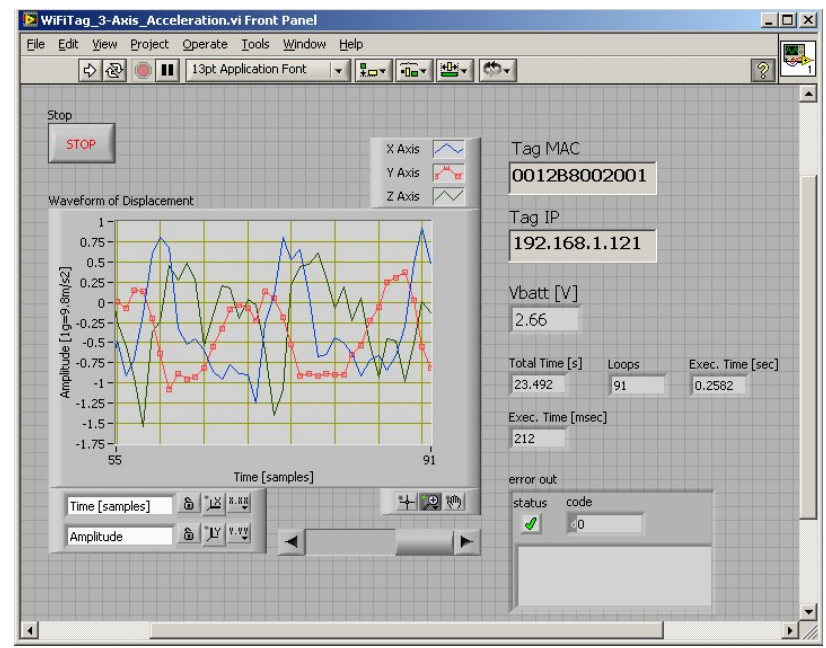

Figure 3. LabVIEW Application 


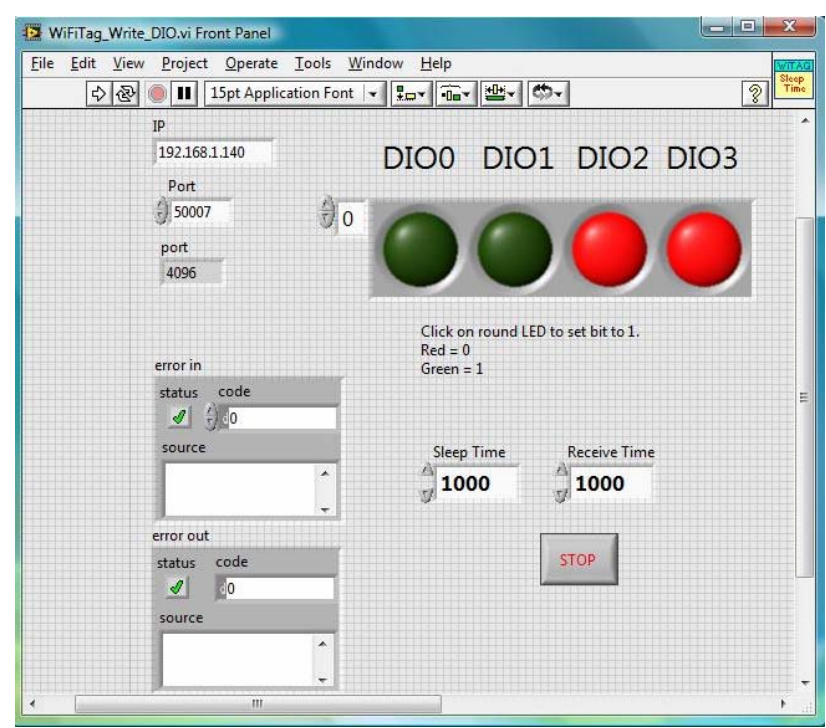

Figure 4. DIO LabVIEW Control

digital I/O lines that control relays or any other devices that require a TTL-level On/Off signal. Thus, the device can run small control algorithms directly or it can respond to commands from larger control programs resident on the Internet.

\section{Control and Alarms}

The following LabVIEW program (Figure 4), based on the Tag4M LabVIEW driver, monitors and writes the four DIO lines. The user first connects TTL-level signals to the Tag4M J3 connector block. Next, power up the tag, establish communication with the AP and then run the LabVIEW program WiFiTag_Read_DIO VI. This VI returns the state of DIO 0,1, 2 and 3 as an I32 integer.

Using an algorithm that users load into the (SPI)EEPROM, the tag can respond to alarm conditions by writing to DIO lines and this must be executed locally without the assistance of a PC. Every wake-up period, the tag executes the code sequence programmed in its firmware. Further, at the end of each wake-up period (following the immediate execution of a complete firmware loop to take readings and issue control signals), the tag asks the wireless access point if there are any incoming commands that it needs to register for execution during the next wake-up period. For instance, the simple opcode (1156, $\mathrm{N})$ reads from or writes to digital line DIO0.

In this way, a web-based control program or the operator - located anywhere with access to the Internet, even over an iPhone - can interactively add commands or modify an existing algorithm. Based on operator inputs, the new combination of firmware code is then executed upon each wake-up.

Moreover, in the future, operators will be able to download entire control algorithms over a WiFi link. This will allow anyone to update or replace the tag firmware without the need to connect the tag to a JTAG connector as is now required.

\section{CONCLUSIONS}

Our paper try to introduce a small price wireless tool with many measurement and monitoring perspectives together with the new and original concept of "WEB INSTRUMENTATION" like a wonderful solution in Predictive Maintenance and Machine Control.

It is easy to understand that this system do not use a special connectivity, only a normal "Access Point" now available in many industrial plants, and it is easy to be installed and configured.

Using LabVIEW drivers and one web page to monitor the system can be easy integrated in the industrial environment. LabVIEW offer the flexibility to develop new applications and the idea of the "WEB INSTRUMENTATION” add the possibility to long distance monitoring and event signalization.

Concluding we proved that this TAG4M system can be considered like a good future solution in Predictive Maintenance and Machine Control.

\section{REFERENCES}

[1] Sabrie Soloman (1998) Sensors Handbook, McGraw-Hill, New York, NY

[2] Jacob Fraden (2004) Handbook of Modern Sensors Physics, Designs, and Applications, Springer-Verlag New York, Inc., NY

[3] www.tag4m.com

[4] M. Ghercioiu, S. Folea, "Ultra-low power Wi-Fi tag for wireless sensing”, IEEE International Conference, Cluj-Napoca, Romania May 22-25, 2008.

[5] D. Ursutiu, P. Cotfas, C. Samoila, "Creativity in Remote Laboratory and Virtual Instrumentation", International Conference on Technology, Communication and Education (i-TCE), April 7-9, 2008, Kuwait

[6] D. Ursutiu, M. Ghercioiu, C. Samoila, "WiFi Sensor for Greenhouse Monitoring”, Int.Conference "Bioatlas”, 4-7 June, 2008, Brasov, Romania

[7] D. Ursutiu, P. Cotfas, C. Samoila, “Online Engineering in University Environment” in "Online Engineering” N. Gupta, S. Ray and T. Sobh, Nova Science Publishers, Inc., 2009, pp.122-160.

\section{AUTHORS}

D.Ursutiu is with the University "Transylvania" from Brasov - Romania and manages the Creativity Laboratory (e-mail: udoru@unitbv.ro).

M. Ghercioiu was with National Instruments USA. He is now with Tag4M Inc., Texas USA (e-mail: info@tag4m.com).

R. Oros prepare her PhD in University "Transylvania” from Brasov - Romania, Creativity Laboratory (e-mail: oros_ramona@yahoo.com).

C.Samoila is with the University "Transylvania" from Brasov - Romania and manages the CVTC Center (email: csam@unitbv.ro).

Manuscript received 05 January 2010. Published as resubmitted by the authors 20 January 2010. 